\title{
Improved Relative Range Equation in Squint SAR for Moving Targets Refocusing
}

\author{
Pu Cheng ${ }^{1}$, ZhentaoYu ${ }^{1 *}$, Jie Chen ${ }^{1}$

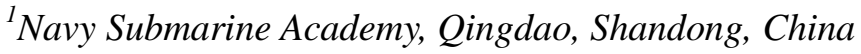 \\ *Corresponding Author.
}

\begin{abstract}
Moving target detection is difficult for synthetic aperture radar (SAR). As SAR is designed for imaging of stationary ground scene, the moving targets would be blurred and displaced in conventional SAR imaging. To increase the signal clutter ratio, the moving targets should be refocused while detecting. Based on relative range equation, one can refocus and detect the moving targets simultaneously by searching the relative velocity. This method has been derived and applied for side looking SAR. In this paper, we extend the relative range equation to squint mode. The procedures of the refocusing method are also illustrated. By introducing a parameter of relative squint angle, the imaging position of the moving target is derived. The refocusing method is validated by simulations. The moving target can be optimally refocused, and the refocused position can be parametrized by the relative motion parameters.
\end{abstract}

Keywords: Equivalent range equation, moving targets, synthetic aperture radar, squint mode

\section{Introduction}

In the area of synthetic aperture radar (SAR) imaging, it is interesting to be able to focus the moving targets [1-3]. As SAR is designed for imaging of stationary scene, the moving targets are blurred and displaced in conventional SAR imaging. The targets would easily migrate several range or azimuth bins, especially for high-resolution SAR or fast-moving targets. Even the transmit signal frequency rate and the Doppler rate are exactly matched, the moving targets would be still defocused. To exactly refocus the moving target, not only the motion parameters but also the position parameters should be considered in the refocusing method.

To resolve this problem, the refocusing method based on SAR imaging is introduced [4-7]. The moving target can be optimally refocused at a fixed position. In the processing, a set of assumption are needed. The moving target is assumed to be relatively static and fixed, and the radar platform is moving constantly. The parameters of the refocusing method are determined by relative motion parameters. Ultimately, the target is refocused at novel position.

First of all, the range history of the moving targets should be exactly modeled. For side looking SAR, a relative exact range equation has been derived[8]. Here, we extend the relative range equation model to squint-mode. A detailed description of the novel model is presented. The procedures of the refocusing method are also illustrated in spatial frequency domain by using the imaging method of wavenumber domain algorithm. At last, the derived model and the refocusing method are validated by simulation results.

ISSN: 0010-8189

(C) CONVERTER 2020

www.converter-magazine.info 


\section{Improved Relative Range Equation}

For slowly moving targets, the slant range between the radar and the moving target is simply expanded as a quadratic Maclaurin series [2].However, if we use this range model, it is hard to totally compensate the range smear and the azimuth smear. Although we can refocus the moving target, the radial velocity and the cross-range velocity have to be searched and completely matched. Furthermore, the range modelin[2] neglects high order terms of the Maclaurin series, and the refocusing result is not optimal. To exactly focus the target and apply the frequency domain methods, an accurate signal model called relative range equation was introduced [4]. Using the derived parameter of relative factor, detecting and refocusing of the moving targets can be simultaneously accomplished.

Assuming a target is moving constantly, its along-track velocity is $v_{\xi}$, and its cross-track velocity is $v_{\eta}$. At time $t_{0}$, it moves to the position $\left(\xi_{0}, \eta_{0}\right)$,so its coordinates $[\xi(t), \eta(t)]$ can be written as

$$
\begin{aligned}
& \xi(t)=v_{\xi}\left(t-t_{0}\right)+\xi_{0} \\
& \eta(t)=v_{\eta}\left(t-t_{0}\right)+\eta_{0}
\end{aligned} .
$$

Assuming the platform fly rectilinearly with a constant speed $v_{p}$, the position of the radar can be written as $x(t)=v_{p} t$.The range between the target and the radar takes on the form

$$
R(t)=\sqrt{[x(t)-\xi(t)]^{2}+\eta^{2}(t)} .
$$

At time $t_{0}$, the moving target moves to the position $\left(\xi_{0}, \eta_{0}\right)$. For convenience, it is assumed that at time $t_{0}$, the moving target happens to be illuminated by the beam center of the radar. This is different with the time $t_{0}$ in [4] and [5] where it denotes the minimum slant range time. Considering asquint SAR with a squint angle $\phi_{q}$, the geometry of the squint SAR is illustrated in Fig.1.The coordinates $\left(\xi_{0}, \eta_{0}\right)$ and $v_{p} t_{0}$ conform to the relationship $\xi_{0}=v_{p} t_{0}+\eta_{0} \tan \phi_{q}$.

Applying the coordinates in (1) to equation (2), like the broadside mode, a similar slant range expression can be derived

$$
\begin{aligned}
& R(t)=\sqrt{\left[v_{p} t-\left(v_{\xi} t-v_{\xi} t_{0}+\xi_{0}\right)\right]^{2}+\left(v_{\eta} t-v_{\eta} t_{0}+\eta_{0}\right)^{2}} \\
& =\sqrt{\left[\left(v_{p}-v_{\xi}\right)^{2}+v_{\eta}^{2}\right] t^{2}+2\left[\left(v_{p}-v_{\xi}\right)\left(v_{\xi} t_{0}-\xi_{0}\right)+v_{\eta}\left(-v_{\eta} t_{0}+\eta_{0}\right)\right] t+\left(v_{\xi} t_{0}-\xi_{0}\right)^{2}+\left(-v_{\eta} t_{0}+\eta_{0}\right)^{2}} . \\
& =\sqrt{\gamma^{2}\left[x(t)-x_{s}\right]^{2}+y_{s}^{2}}
\end{aligned}
$$

The factor $\gamma$ is a key parameter

ISSN: 0010-8189 


$$
\gamma=\sqrt{\left(1-u_{\xi}\right)^{2}+u_{\eta}^{2}}, \quad u_{\xi}=\frac{v_{\xi}}{v_{p}}, \quad u_{\eta}=\frac{v_{\eta}}{v_{p}} .
$$

It combines the cross-track velocity and the along-track together. The factor $\gamma$ can be considered as the normalized amplitude of the relative speed. We call it relative velocity factor. The expression (4) is the same with the broadside mode [8]. The coordinates $\left(x_{s}, y_{s}\right)$ represent the refocused position

$$
x_{s}=\xi_{0}-\frac{u_{\eta}}{\gamma^{2}} \eta_{0}+\frac{1-\gamma^{2}-u_{\xi}}{\gamma^{2}} \eta_{0} \tan \phi_{q}, \quad y_{s}=\frac{1-u_{\xi}+u_{\eta} \tan \phi_{q}}{\gamma} \eta_{0} .
$$

The moving target will be ultimately refocused at this position. The expression of the refocused position is different from the broadside mode [8]. It can be seen from (3) that the variable $y_{s}$ denotes the minimum relative slant range.

It should be noted that, in the above derivation, the velocity of the moving target is assumed to be constant. This assumption is usually sufficient for ground moving targets. Approximately, an accelerating target can be regarded as a constantly moving target in the synthetic aperture duration.

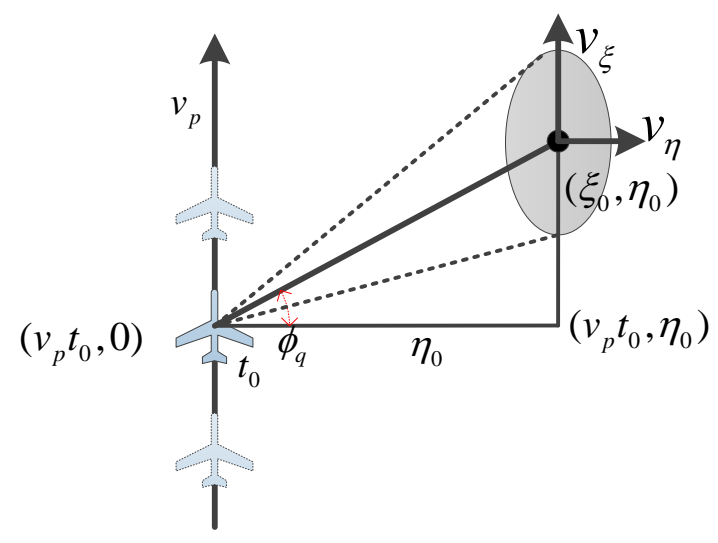

Fig. 1: Geometry of the squint SAR with a squint angle $\phi_{q}$. At beam center crossing time $t_{0}$, the target is located at the position $[\xi(t), \eta(t)]=\left(\xi_{0}, \eta_{0}\right)$, and the platform at $[x(t), 0]=\left(v_{p} t_{0}, 0\right)$.

\section{Relative Squint Angle}

\subsection{Relative Squint Angle}

As shown in Fig. 2, we define the angle difference between the real direction of the platform and the relative direction of the platform as relative squint angle $\phi_{r}$

ISSN: 0010-8189 


$$
\cos \phi_{r}=\frac{1-u_{\xi}}{\gamma}, \quad \sin \phi_{r}=\frac{u_{\eta}}{\gamma} .
$$

The real or original squint angle is $\phi_{q}$. A positive value of the cross-track velocity $u_{\eta}$ results to the subtraction of $\phi_{r}$ from $\phi_{q}$, i.e. the equivalent squint angle $\phi_{e}$ is

$$
\phi_{e}=\phi_{q}-\phi_{r}
$$

Using the relative squint angle, the refocused position (4)can be rewritten in another form

$$
x_{s}=\xi_{0}-\frac{1}{\gamma} \eta_{0} \sin \phi_{r}+\frac{1}{\gamma} \eta_{0} \tan \phi_{q}\left[u_{\xi} \cos \phi_{r}-u_{\eta} \sin \phi_{r}\right], \quad y_{s}=\eta_{0} \frac{\cos \left(\phi_{q}-\phi_{r}\right)}{\cos \phi_{q}}=\eta_{0} \frac{\cos \phi_{e}}{\cos \phi_{q}}
$$

For broadside mode $[8],\left(x_{s}, y_{s}\right)$ reduce to

$$
x_{s}=\xi_{0}-\frac{1}{\gamma} \eta_{0} \sin \phi_{r}, \quad y_{s}=\eta_{0} \cos \phi_{r}
$$

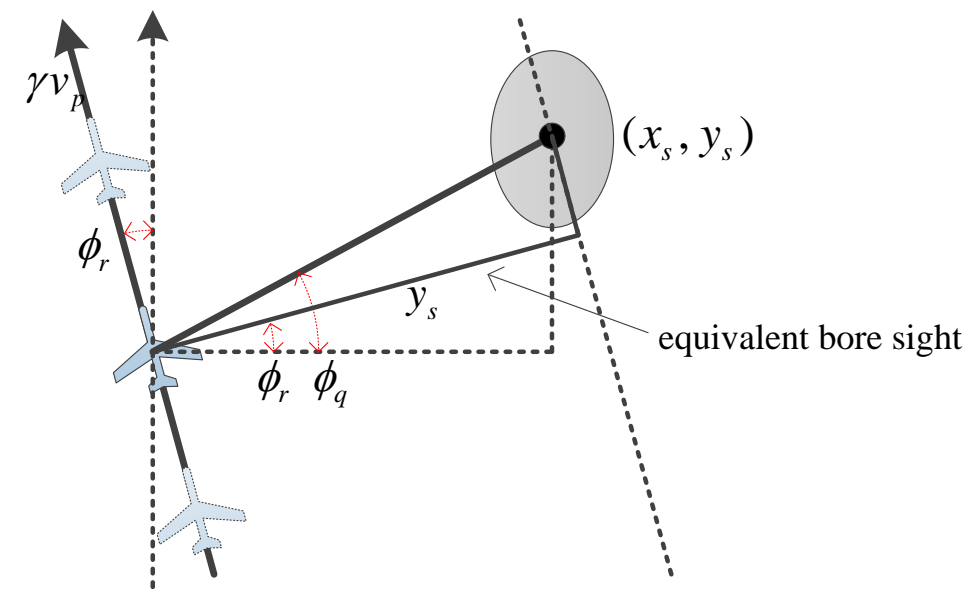

Fig.2: The radar platform is moving rectilinearly with a relative speed. The magnitude of the relative speed is $\gamma v_{p}$. The relative squint angle is $\phi_{r}$.

3.2 Equivalent Scan Angle and Doppler Frequency

Like the scan angle in conventional SAR imaging, we define a novel angle called equivalent scan angle $\theta$

$$
\cos \theta=\frac{y_{s}}{R(t)}, \quad \sin \theta=\frac{\gamma\left(x_{s}-v_{p} t\right)}{R(t)}
$$

ISSN: 0010-8189 
It can be regarded as the angle between radar target sight line and equivalent bore sight line which is perpendicular to the relative speed shown in Fig.2. The Doppler frequency of the moving target can be presented by angle $\theta$

$$
f_{d}=-\frac{2}{\lambda} \times \frac{\mathrm{d} R(t)}{\mathrm{d} t}=\frac{2 \gamma v_{p}}{\lambda} \sin \theta
$$

where $\lambda$ represents the radar operating wavelength. The equivalent scan angle can be regarded as the angle between radar-target line and the equivalent bore sight shown in Fig. 2.

It can also be seen from Fig. 2 that the Doppler centroid of the Doppler frequency is the Doppler frequency at time $t_{0}$, and at this time, the equivalent scan angle $\theta$ equals to the equivalent squint angle $\phi_{q}-\phi_{r}$.

$$
f_{d c}=\frac{2 \gamma v_{p}}{\lambda} \sin \left(\phi_{q}-\phi_{r}\right)=\frac{2 \gamma v_{p}}{\lambda} \sin \phi_{e}
$$

So, the Doppler bandwidth is

$$
f_{d} \in\left[\frac{2 \gamma v_{p}}{\lambda} \sin \left(\phi_{e}-\frac{\theta_{B W}}{2}\right), \frac{2 \gamma v_{p}}{\lambda} \sin \left(\phi_{e}+\frac{\theta_{B W}}{2}\right)\right]
$$

in which $\theta_{B W}$ is the antenna beamwidth. From (13), we can see the frequency bandwidth is determined by the relative speed, equivalent squint angle and the beamwidth.

\subsection{Amplitude and Angle of the Relative Velocity}

The relative factor $\lambda$ and the relative squint angle $\phi_{r}$ respectively denote the magnitude and the direction of the relative velocity. The factor $\gamma$ is mainly affected by the along-track velocity $v_{\xi}$. The relative squint angle $\phi_{r}$ is mainly affected by the cross-track velocity $u_{\eta}$. It can be easily obtained from the partial derivatives of (3) and (5) that

$$
\begin{gathered}
\left|\frac{\partial \gamma}{\partial u_{\xi}}\right|=\left|\frac{1-u_{\xi}}{\gamma}\right|, \quad\left|\frac{\partial \gamma}{\partial u_{\eta}}\right|=\left|\frac{u_{\eta}}{\gamma}\right| \\
\left|\frac{\partial \sin \phi_{r}}{\partial u_{\xi}}\right|=\left|\frac{u_{\eta}\left(1-u_{\xi}\right)}{\gamma^{3}}\right|, \quad\left|\frac{\partial \sin \phi_{r}}{\partial u_{\eta}}\right|=\left|\frac{\left(1-u_{\xi}\right)^{2}}{\gamma^{3}}\right| .
\end{gathered}
$$

As the platform velocity is usually high, $\left|1-u_{\xi}\right|>\left|u_{\eta}\right|$.We can see from (14) and (15) that the factor $\gamma$ is much ISSN: 0010-8189 
more affected by the along-track velocity, and the angle $\phi_{r}$ is much more affected by the cross-track velocity $v_{\eta}$.

\section{Refocusing and Simulations}

The SAR system moves along the flight path transmitting and receiving the radar pulses. The received signal is demodulated by a replica of the transmitted signal. Here, a point scatter is simulated. The intermediate frequency signal [9] after removal of residual video phase is

$$
s_{\mathrm{if}}(t)=\exp \left[-\mathrm{j} 2 \pi\left(f_{c}+k_{r} t_{r}\right) \times \frac{2 R(t)}{c}\right]
$$

in which $f_{c}$ denotes the operating or carrier frequency, and $k_{r}$ denotes the frequency rate. $t_{r}$ is the fast time or range time. By neglecting the fast time, the range curvature $R(t)$ takes on the form

$$
R\left(t_{a}\right) \approx \sqrt{\gamma^{2}\left(v_{p} t_{a}-x_{s}\right)^{2}+y_{s}^{2}} .
$$

where $t_{a}$ is the slow time or azimuth time.

\subsection{Refocusing Method}

Perform Fourier transform to the signal (14) with respect to the azimuth position $x\left(t_{a}\right)=v_{p} t_{a}$. A wavenumber domain signal is obtainedby using the stationary phase principle

$$
S_{\text {if }}\left(K_{x}, K_{R}\right)=\exp \left(-\mathrm{j} K_{x} x_{s}\right) \exp \left(-\mathrm{j} y_{s} \sqrt{K_{R}^{2}-\frac{K_{x}^{2}}{\gamma^{2}}}\right)
$$

in which $K_{R}$ is the range wave number, and $K_{x}$ is the azimuth wave number

$$
\begin{aligned}
& K_{R}=\frac{4 \pi}{c}\left(f_{c}+k_{r} t_{r}\right) \\
& K_{x}=\frac{2 \pi f_{d}}{v_{p}}=\frac{4 \pi \gamma \sin \theta}{\lambda} .
\end{aligned}
$$

Thus, we can refocus the moving target using conventional SAR imaging algorithms like the wavenumber domain algorithm or others. The difference is that the relative velocity factor must be considered in imaging procedures.

\subsection{Simulation Results}

To validate our derivation and the refocusing method, a stationary target and a moving target located at the same

ISSN: 0010-8189

(C) CONVERTER 2020 
position are simulated. At time $t_{0}$, the platform is located at $\left(v_{p} t_{0}, 0\right)=(600 m, 0 m)$. The squint angle is $30 \mathrm{deg}$, and both the two targets are located at $\left(\xi_{0}, \eta_{0}\right)=(637.25 m, 1000 m)$. The pulse repetition frequency of the SAR is $f_{p}=500 \mathrm{~Hz}$ and the carrier frequency is $f_{c}=10 \mathrm{GHz}$. The velocity of platform is $v_{p}=50 \mathrm{~m} / \mathrm{s}$. The along-track and cross-track velocities of the moving target are respectively $v_{\xi}=3 \mathrm{~m} / \mathrm{s}$ and $v_{\eta}=2 \mathrm{~m} / \mathrm{s}$. That is the relative factor is $\gamma=0.94$.

After conventional SAR imaging, as shown in Fig. 3, the static target is focused and located at $\left(\xi_{0}, \eta_{0}\right)$, and the moving target is defocused and shifted along azimuth. In this figure, the red dashed lines illustrate the boresight of 30 deg and the perpendicular of the boresight direction. We can see that the range and azimuth direction of the static target go well with the red dashed line.

After refocusing by using the method presented above, the moving target is refocused and located at $(627.8 m, 1023.6 m)$. It is shown in Fig. 4. The relative squint angle $\phi_{r}$ of the moving targets can be calculated by using (6). It is about $2.4 \mathrm{deg}$. Thus, the equivalent squint angle $\phi_{e}$ is about $27.6 \mathrm{deg}$. The red dashed lines are the same with Fig.3. They illustrate the boresight with an angle of 30deg and its perpendicular. The moving target in Fig.4 is zoomed in Fig.5. We can see the angle difference between the boresight and the range direction of the refocused moving target. This angle difference is the relative squint angle. We can also see that the stationary target is blurred. The simulated results validate our refocusing method and analysis.

\section{Conclusion}

By using relative range equation model, moving targets can be optimally refocused. In this paper, we extend this model to squint mode. By taking the relative squint angle into consideration, we extend the conventional wavenumber domain algorithm in SAR imaging to refocus moving targets. High range resolution and azimuth resolution like the SAR imaging can be obtained This is very attractive for SAR system boasting ground moving target indication. Simulations validate our derivation and the refocusing method. The simulated moving target is refocused at the right place expressed by the relative factor and the relative squint angle.

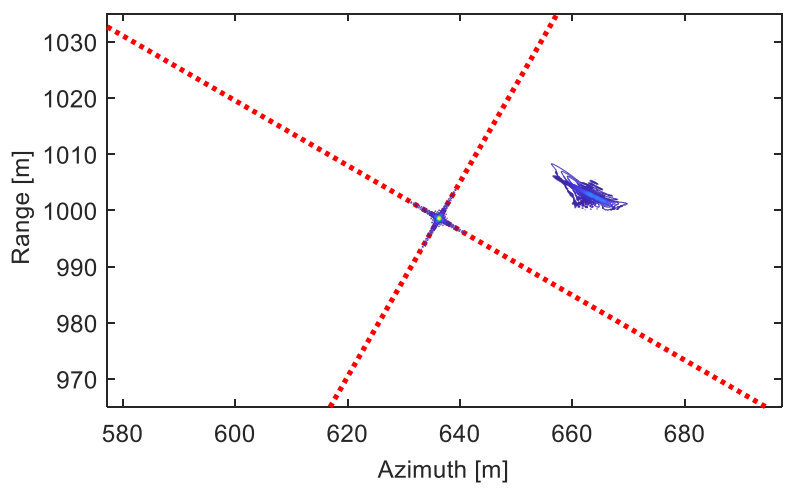

Fig.3: Imaging of a static target and a moving target both located at $(637.25 m, 1000 m)$ in a squint SAR. The

ISSN: 0010-8189

(C) CONVERTER 2020 
moving target is blurred and shifted in the SAR image.

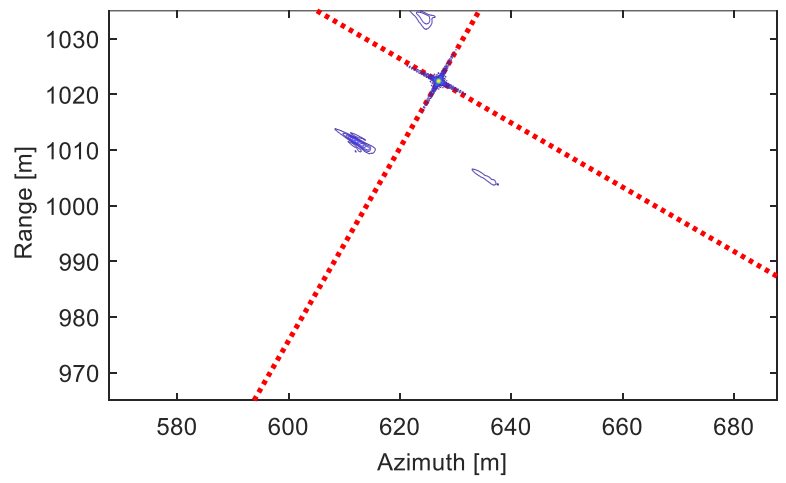

Fig.4: After refocusing by using the presented method, the moving target is refocused. It is located at a novel position $\left(x_{s}, y_{s}\right)=(627.8 m, 1023.6 m)$.The static target is blurred.

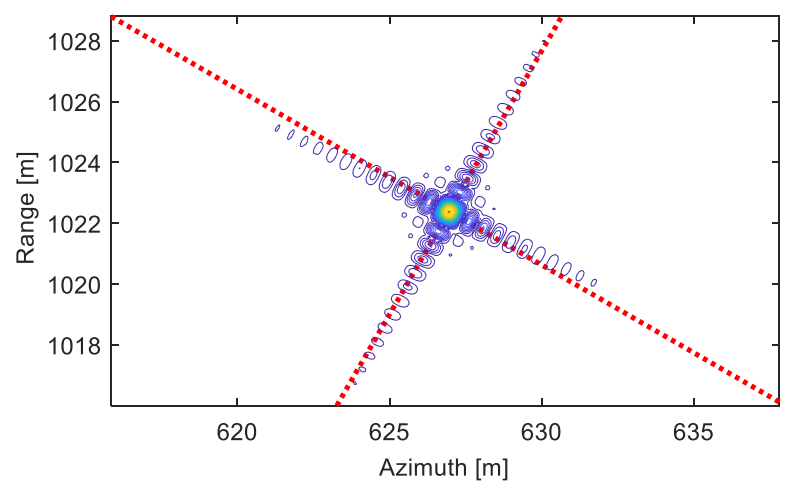

Fig.5: A zoomed figure of the refocused moving target. The red dashed lines illustrate the boresight angle of 30 deg. The target is refocused and its relative squint angle is $2.4 \mathrm{deg}$.

\section{References}

[1] J.K. Jao (2002). Theory of synthetic aperture radar imaging of a moving target. IEEE Transactions on Geoscience and Remote Sensing, 39(9), 1984-1992.

[2] R.K. Raney (1971). Synthetic aperture imaging radar and moving targets. IEEE Transactions on Aerospace and Electronic Systems, AES-7(3), 499-505.

[3] J. R. Fienup (2001). Detecting moving targets in sar imagery by focusing. IEEE Transactions on Aerospace \& Electronic Systems, 37(3), 794-809.

[4] Mehrdad Soumekh (1997). Moving target detection in foliage using along track monopulse synthetic aperture radar imaging. IEEE Transactions on Image Processing.

[5] V.T. Vu, T.K. Sjogren, M.I. Pettersson, et al. (2010). Detection of moving targets by focusing in uwbsar-theory and experimental results. IEEE Transactions on Geoscience \& Remote Sensing, 48(10), 3799-3815.

[6] V.T.Vu, M.I. Pettersson, and T.K. Sjogren (2017). Moving target focusing in sar image with known normalized relative speed. IEEE Transactions on Aerospace and Electronic Systems, 53(2), 854-86.

ISSN: 0010-8189 
[7] Viet Thuy Vu (2018). Local detection of moving targets in sar image based on nrs hypotheses. IEEE Transactions on Geoence \& Remote Sensing, PP, 1-10.

[8] Pu Cheng, Qin Xin, Jian Wei Wan and Zhan Wang (2015). Efficient detection of ground moving targets in FMCW SAR by focusing. IEEE Geoscience \& Remote Sensing Symposium.

[9] Y. Liang, Y. Huai, J.Ding, H. Wang and M. Xing. (2016). A modified $\omega-k$ algorithm for hs-sar smallaperture data imaging. IEEE Transactions on Geoscience and Remote Sensing, 54(6), 3710-3721.

ISSN: 0010-8189 